\title{
Parton Distributions: Progress and Challenges
}

\author{
Andreas Vogt \\ Department of Mathematical Sciences, University of Liverpool \\ Liverpool L69 3BX, United Kingdom
}

\begin{abstract}
We briefly discuss recent research on the spin-averaged parton densities of the proton, focusing on some aspects relevant to hard processes at the LHC. Specifically, after recalling the basic framework and the need for higher-order calculations, we address the evolution equations governing the scale dependence of the parton distributions and their solution, schemes for initial conditions and the inclusion of heavy quarks, recent progress on fits to data, and future high-precision constraints from LHC measurements.
\end{abstract}

\section{Introduction: partons for the LHC}

For at least the next ten years, proton-(anti-) proton colliders will continue to form the high-energy frontier in particle physics. At such machines, many quantitative studies of hard (high mass/scale) standard-model and new-physics processes require a precise understanding of the parton structure of the proton. The present talk [1] briefly discusses some recent developments in this field.

We start by recalling the description of hard proton processes using the simplest case, inclusive lepton-proton deep-inelastic scattering (DIS), the process providing the major part of the present constraints on the parton densities. Here the hard scale is the virtuality $Q^{2}=-q^{2}$ of the exchanged gauge boson, a photon in Fig. 1, and the Bjorken variable $x=Q^{2} /(2 P q)$, with $P$ the proton momentum, is usually chosen as the second independent variable. At zeroth order in the strong coupling constant $\alpha_{\mathrm{s}}$ the hard coefficient functions $c_{a, i}$ are trivial, and the momentum fraction $\xi$ carried by the struck quark $i$ is equal to Bjorken- $x$ if mass effects are neglected.

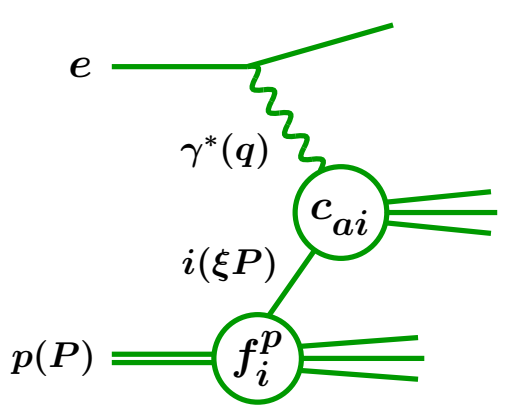

Figure 1: Kinematics of photon-exchange DIS in the QCD-improved parton model. Particle momenta are given in brackets.

In general, the structure functions $F_{2, L}^{p}$ for the process of Fig. 1 are given by

$$
x^{-1} F_{a}^{p}\left(x, Q^{2}\right)=\sum_{i=q, g} \int_{x}^{1} \frac{d \xi}{\xi} c_{a, i}\left(\frac{x}{\xi}, \alpha_{\mathbf{s}}\left(\mu^{2}\right), \frac{\mu^{2}}{Q^{2}}\right) f_{i}^{p}\left(\xi, \mu^{2}\right)
$$

plus terms of order $1 / Q^{2}$ which, for the purpose of high-scale predictions, are best suppressed by sufficiently stringent cuts on the fitted experimental data. Besides on the factorization scheme used to define the parton densities $f_{i}^{p}$ - in this talk $\overline{\mathrm{MS}}$ unless stated otherwise the coefficient functions depend on the renormalization and factorization scale $\mu$ (identified here for notational simplicity) which ought to be chosen as $\mu^{2}=\mathcal{O}\left(Q^{2}\right)$ in order to avoid large logarithms. The parton distributions depend on this scale via the evolution equations

$$
\frac{d}{d \ln \mu^{2}} f_{i}\left(\xi, \mu^{2}\right)=\sum_{k}\left[P_{i k}\left(\alpha_{\mathrm{s}}\left(\mu^{2}\right)\right) \otimes f_{k}\left(\mu^{2}\right)\right](\xi) .
$$


Here $\otimes$ is a short-hand for the Mellin convolution written out in Eq. (1) above. The initial conditions for Eq. (2) are, of course, not calculable in perturbative QCD. As lattice results are restricted to very few Mellin moments (with, at present, still rather limited accuracy), predictions for collider cross sections are obtained via fits to suitable sets of reference observables, including structure functions in DIS, and the universality of the parton densities.

The splitting functions $P$ and the process-dependent hard coefficient functions $c_{a}$ admit expansions in powers of $\alpha_{\mathrm{s}}$,

$$
\begin{aligned}
P & =\alpha_{\mathrm{s}} P^{(0)}+\alpha_{\mathrm{s}}^{2} P^{(1)}+\alpha_{\mathrm{s}}^{3} P^{(2)}+\ldots \\
c_{a} & =\alpha_{\mathrm{s}}^{n_{a}}\left[c_{a}^{(0)}+\alpha_{\mathrm{s}} c_{a}^{(1)}+\alpha_{\mathrm{s}}^{2} c_{a}^{(2)}+\ldots\right]
\end{aligned}
$$

with, for example, $n_{a}=0$ for $F_{2}$ and $n_{a}=1$ for $F_{L}$. For a consistent approximation the same number of terms has to be kept in the two lines of Eq. (3). The first $n+1$ terms define the $\mathrm{N}^{\mathrm{n}} \mathrm{LO}$ approximation. As the normalization of the LO prediction is rather arbitrary, the next-to-leading order (NLO) provides the first real prediction of the cross sections and, consequently, the NNLO the first serious error estimate of the perturbative expansions.

The successive approximations of perturbative QCD are illustrated in Fig. 2 for an LHC process of utmost importance, the production of the standard-model Higgs boson dominated by gluon-gluon fusion via a top-quark loop. Obviously the NLO approximation [2] is insufficient for a quantitative prediction in this case, and even at NNLO [3,4] higher-order uncertainties of about $15 \%$ remain for the total cross section. A perturbative accuracy of $5 \%$ is only reached at $\mathrm{N}^{3} \mathrm{LO}$, known to a sufficient approximation from Ref. [5] (for an extension to the rapidity distribution see Ref. [6]). Note that these uncertainties do not include those of the coupling $\alpha_{\mathrm{s}}$ and the parton densities, taken for Fig. 2 from Ref. [7] where at NNLO previous (but sufficiently accurate) approximations [8] were used for $P^{(2)}$ in Eq. (3).
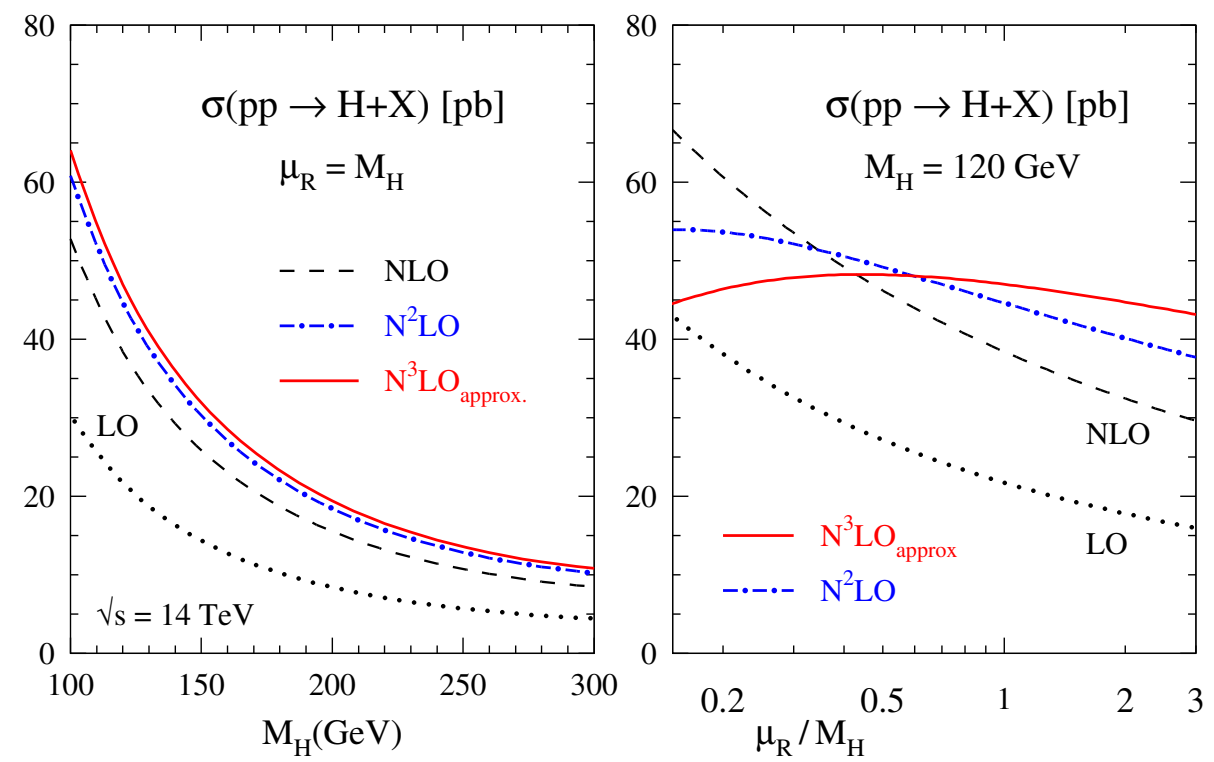

Figure 2: Perturbative expansion of the total cross section for Higgs production at the LHC. Shown are the dependence on the mass $M_{H}$ and the renormalization scale $\mu_{\mathrm{r}}$ (from Ref. [5]). 
The minimal momentum fractions $\xi_{-}$of partons contributing to the production of a particle of mass $M$ at the LHC are shown in Fig. 3, together with the kinematic reach of HERA and fixed-target DIS experiments at the corresponding scales $Q^{2}$. Taking into account also the limited rapidity coverage of the LHC detectors, one reads off $\xi_{-} \gtrsim 10^{-4}$ for the most important processes, including the production of the $W, Z$ and Higgs bosons and the top quark, and the search for new particles. Thus the HERA data can be fitted with a cut of $Q^{2} \approx 10 \mathrm{GeV}^{2}$ which should be sufficient to suppress low-scale instabilities (as, e.g., in $F_{L}$ to NNLO [9]) and power corrections to Eq. (1).

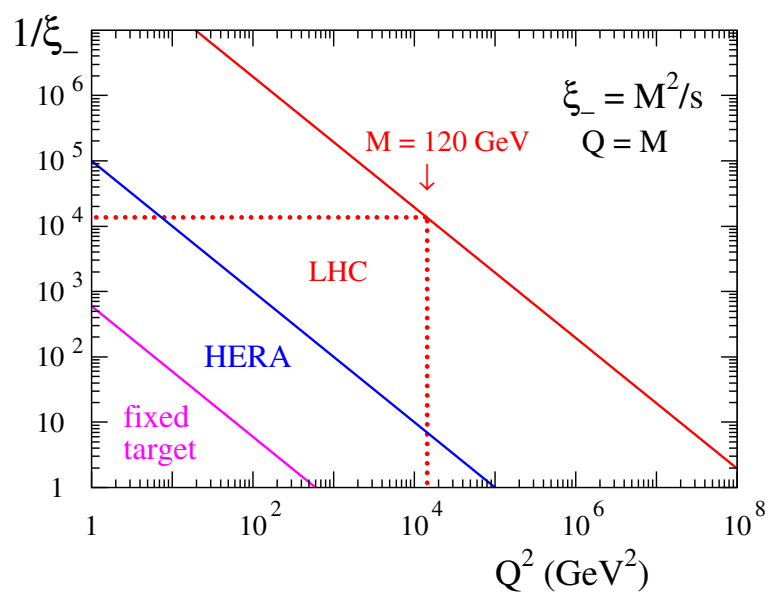

Figure 3: Minimal parton momenta $\xi_{-}$probed at the LHC, compared with the DIS coverage of HERA and previous fixed-target experiments.

\section{Higher orders in the parton evolution}

The complete NNLO splitting functions $P^{(2)}(x)$ - from now on we, as usual, denote also the parton momentum fractions by $x$ - in Eq. (3) have been computed three years ago in Refs. [10,11]. We first consider the flavour non-singlet evolution of quark-distribution differences such as the combination $q_{\mathrm{ns}}^{+}=u+\bar{u}-(d+\bar{d})$ probed by $F_{2}^{p}-F_{2}^{n}$. Figure 4 illustrates the perturbative expansion of the Mellin moments of the corresponding splitting function and the resulting approximations for the scale dependence of $q_{\text {ns }}^{+}$at large $x$.
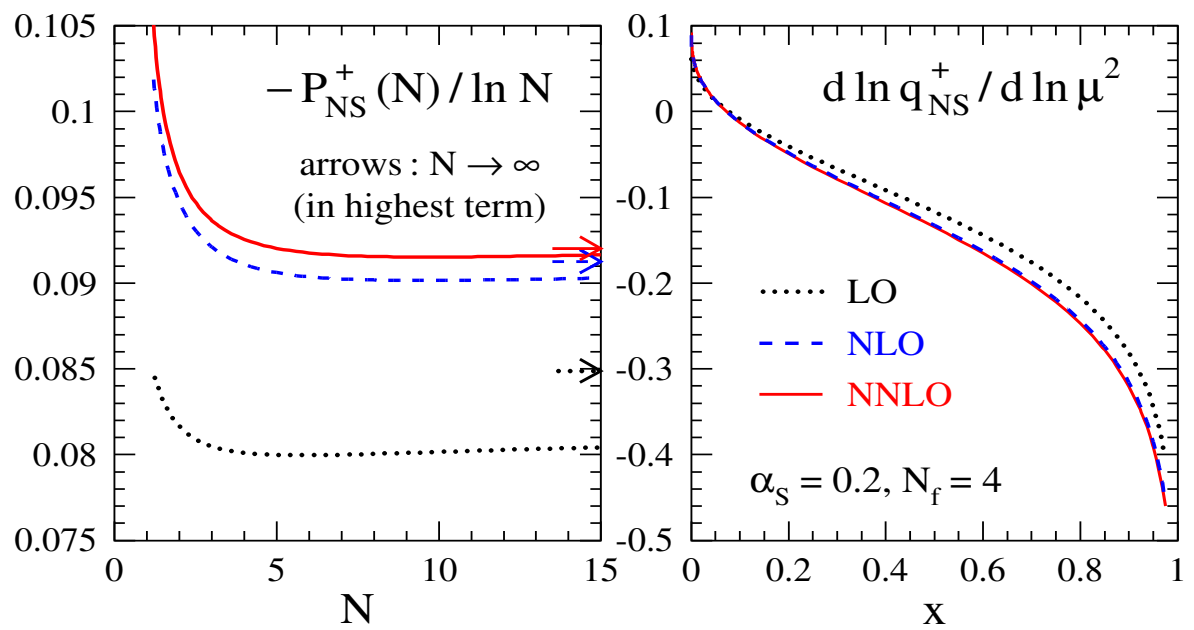

Figure 4: The LO, NLO and NNLO approximations to the splitting-function moments $P_{\mathrm{ns}}^{+}(N)$ for four flavours at $\alpha_{\mathrm{s}}=0.2$, and the resulting logarithmic scale derivatives for $x q_{\mathrm{ns}}^{+}=x^{0.5}(1-x)^{3}$, a schematic but characteristic model distribution (from Ref. [10]). 
The first fourth-order result for this splitting function has been presented last year [12]: the second moment of $P_{\mathrm{ns}}^{+}(x)$ is now known to $\mathrm{N}^{3} \mathrm{LO}$ for three flavours, numerically reading

$$
P_{\mathrm{ns}}^{+}\left(N=2, n_{f}=3\right)=-0.283 \alpha_{\mathrm{s}}\left[1+0.869 \alpha_{\mathrm{s}}+0.798 \alpha_{\mathrm{s}}^{2}+0.926 \alpha_{\mathrm{s}}^{3}+\ldots\right] .
$$

Taking into account the weak $N$-dependence of $P_{\mathrm{ns}}^{+}$at $N>2$ demonstrated in Fig. 4 , this result sets the scale for the $\mathrm{N}^{3} \mathrm{LO}$ contributions for the whole large- $x$ region. According to the general pattern, the corresponding corrections for $n_{f}=4 \ldots 6$ will be even smaller.

The low- $x$ behaviour of the non-singlet splitting functions and coefficient functions is not too relevant in practice, but provides an interesting lab for the study of small- $x$ logarithms: unlike in the singlet case, two additional powers of $\ln x$ enter per order in $\alpha_{\mathrm{s}}$, e.g., terms up to $\ln ^{4} x$ and $\ln ^{5} x$ occur in $P_{\mathrm{ns}}$ and $c_{2, \mathrm{~ns}}$ already at order $\alpha_{\mathrm{s}}^{3}$. Successive approximations of these functions including the leading, next-to-leading, ... small- $x$ terms are shown in Fig. 5. Obviously, a 'low-order' approximation of this type is not appropriate at any $x$-values relevant to colliders. Consequently leading- and next-to-leading-log resummations can, at best, provide only very rough indications of the maximal size of the higher-order corrections. In the present case the all-order leading-logarithmic contributions [13] are small enough to exclude small- $x$ instabilities, e.g., for $x q_{\mathrm{ns}}^{+} \sim x^{0.5}$, down to extremely low values of $x$ [14].
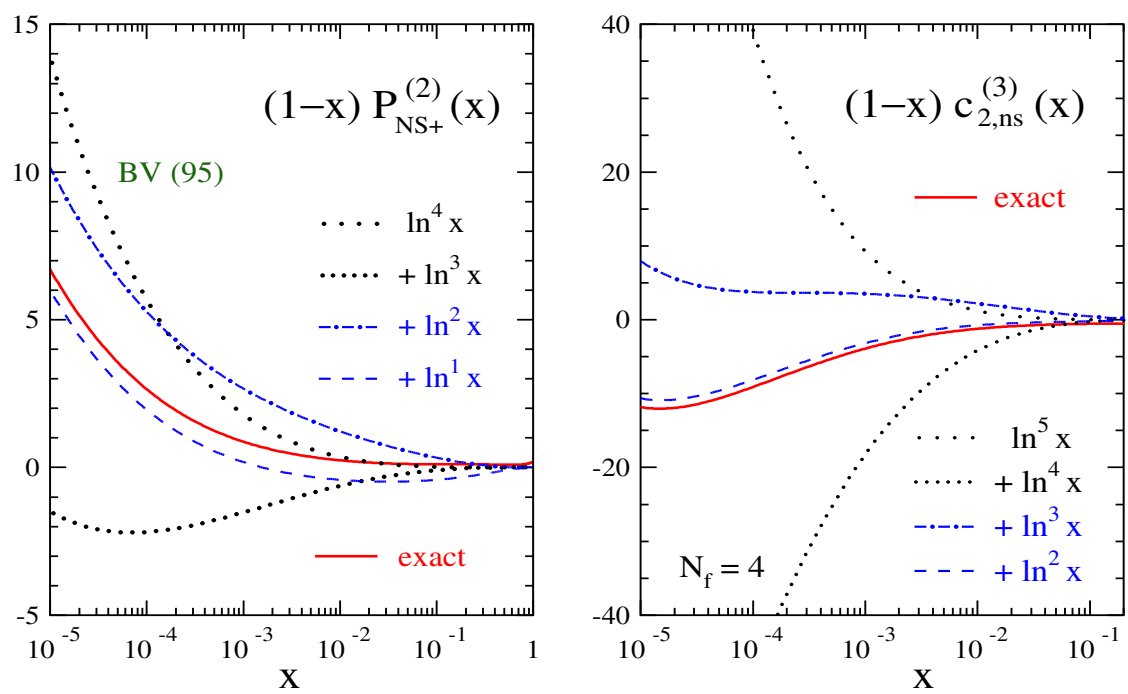

Figure 5: The exact $\alpha_{\mathrm{s}}^{3}$ contributions to the non-singlet splitting function and coefficient function for $F_{2}$, compared to approximations obtained from the small- $x$ logarithms (from Refs. [10,15]). The leading small- $x$ term of $P_{\mathrm{ns}+}^{(2)}$ was derived before in Ref. [13].

The flavour-singlet splitting functions are vital for transferring small- $x$ information from HERA to LHC scales across up to three orders of magnitude in $Q^{2}$, recall Fig. 3 . The corresponding NNLO contribution [11] to Eq. (2) is shown in Fig. 6 for the gluon-gluon case. Also here the leading small- $x$ term, obtained before in Ref. [16] (and transformed to $\overline{\mathrm{MS}}$ in Ref. [17]), does not provide a good approximation for any practically relevant values of $x$. Moreover, the splitting functions enter physical quantities only via the Mellin convolutions of Eqs. (1) and (2). Hence a locally accurate low- $x$ approximation, as provided for $P_{\mathrm{gg}}^{(2)}$ by the $x^{-1} \ln x$ plus the $x^{-1}$ terms at $x \lesssim 10^{-3}$, is insufficient for $d g / d \ln \mu^{2}$ even at small $x$. 

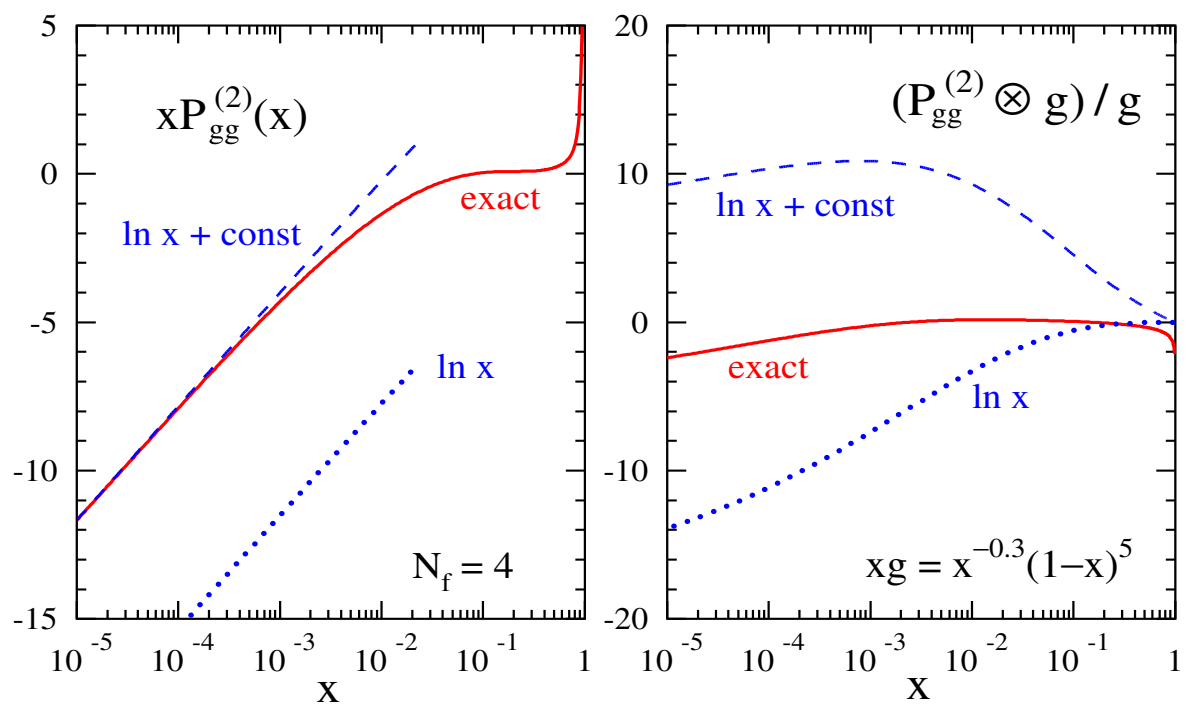

Figure 6: Left: the $\alpha_{\mathrm{s}}^{3}$ contribution $P_{\mathrm{gg}}^{(2)}$ to the gluon-gluon splitting function, compared to its leading $[16,17]$ and next-to-leading small- $x$ approximations. Right: the convolution of these three functions with a schematic but typical gluon distribution (from Ref. [11]).

Consequently, reliable estimates of the post-NNLO corrections to the small- $x$ evolution will become possible, via approximations analogous to those of Ref. [17], only once a few singlet moments have been computed to order $\alpha_{\mathrm{s}}^{4}$. Fortunately, as illustrated in Fig. 7, the expansion of the quark and gluon evolution to NNLO appears to be very stable, at least for the main HERA-to-LHC region $x \gtrsim 10^{-4}$ at $Q^{2} \gtrsim 10 \mathrm{GeV}^{2}$ (see above).
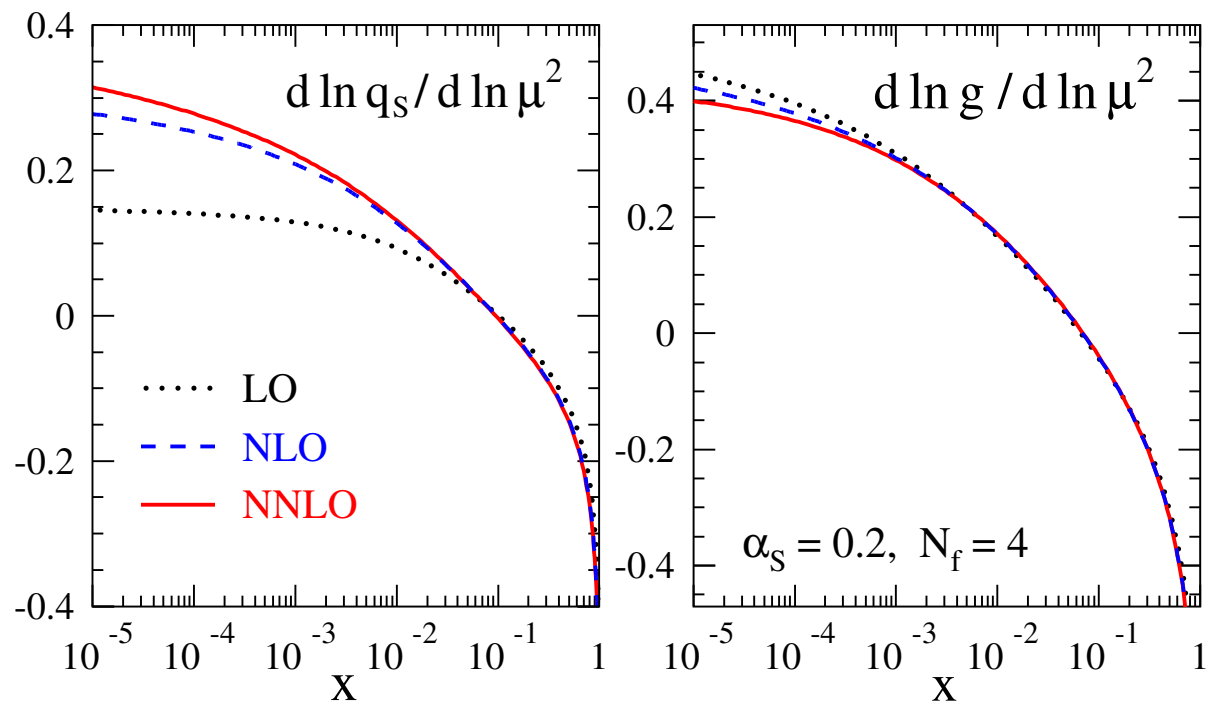

Figure 7: Perturbative expansion of the scale derivatives of typical quark and gluon distributions at $\mu^{2} \approx 30 \mathrm{GeV}^{2}$ (from Ref. [11], where the initial conditions are specified). 


\section{Solutions of the evolution equations}

The most direct manner to solve the system (2) of coupled integro-differential equations is by a discretization in both $x$ and $\mu^{2}$. Recently written or updated public codes including the NNLO evolution are HOPPET [18] and the new version 17 (beta-released at the time of this talk) of QCDNUM [19]. Alternatively, Eqs. (2) can be transformed to ordinary differential equations in (complex) Mellin- $N$ space. These are then treated analytically and the solutions transformed back by quadratures. This approach has been employed in QCD-PEGAsus [20]. The left part of Fig. 8 shows a sample comparison of the programs [18] and [20], using the Les Houches initial conditions discussed below. The right part of the figure, taken from the QCDNUM manual, illustrates the greatly improved numerical accuracy of the new version.
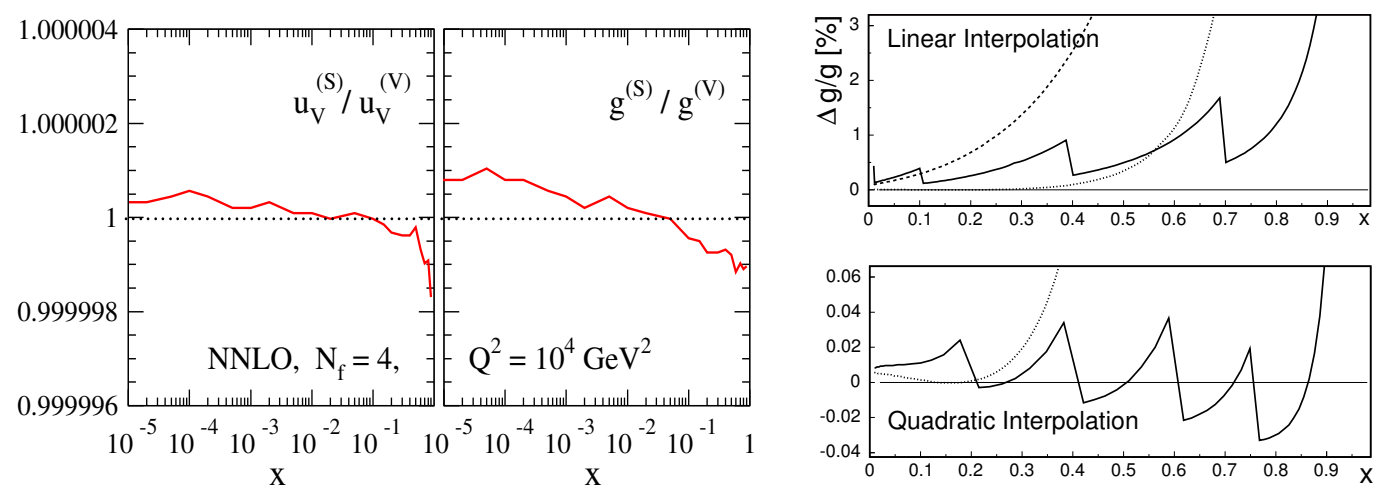

Figure 8: Left: ratios of high-scale NNLO up-valence and gluon distributions after evolution with the codes [18] (S) and [20] (V). Right: the accuracy improvement of QCDNUM due to the new quadratic $x$-interpolation with actually fewer points (for details see Ref. [19]).

Obviously it is very useful, e.g., for validating newly written or ported codes, to have at one's disposal a set of benchmark evolution results. A reference input was set up for this at the 2001 Les Houches collider-physics workshop (see Ref. [21] for the complete expressions),

$$
x u_{\mathrm{v}}\left(x, \mu_{\mathrm{f}, 0}^{2}\right)=5.1072 x^{0.8}(1-x)^{3}, \ldots, x g\left(x, \mu_{\mathrm{f}, 0}^{2}\right)=1.7000 x^{-0.1}(1-x)^{5}
$$

for the initial factorization scale $\mu_{\mathrm{f}, 0}^{2}=2 \mathrm{GeV}^{2}$ and the coupling $\alpha_{\mathrm{s}}\left(\mu_{\mathrm{r}}^{2}=2 \mathrm{GeV}^{2}\right)=0.35$. As illustrated in Fig. 8, the results of the programs [18] and [20] agree to five significant digits over a wide range in $x$ and $\mu_{\mathrm{f}}^{2}$, a level of agreement not reached before between $x$-space and $N$-space programs. The results at the important scale $\mu_{\mathrm{f}}^{2}=10^{4} \mathrm{GeV}^{2}$ have therefore been cast into reference tables for the evolution at LO, NLO - including, for different initial conditions, the spin-dependent case - and NNLO for the scales ratios $\mu_{\mathrm{r}} / \mu_{\mathrm{f}}=0.5,1$ and 2 , using both a fixed and a variable number of flavours $n_{f}$ (see below). For example, the (iterated, see Ref. [20]) four-flavour NNLO evolution of Eq. (5) for $\mu_{\mathrm{f}}=2 \mu_{\mathrm{r}}$ yields

$$
\begin{aligned}
& x=10^{-5}, \quad x u_{v}=2.9032 \cdot 10^{-3}, \quad \ldots, \quad x g=2.2307 \cdot 10^{2}
\end{aligned}
$$

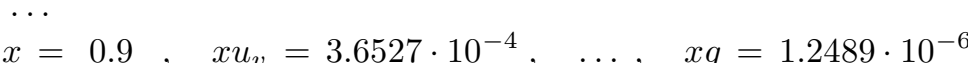

at this scale. The complete tables can be found in Refs. [21]. It would be very reasonable to employ only programs which have been checked against these benchmarks. 


\section{Input shapes, factorization schemes and heavy quarks}

The $\overline{\mathrm{MS}}$ scheme adopted so far is calculationally convenient and leads to a perturbatively stable parton evolution - recall Eq. (4), Figs. 4 and 7. However, the NLO, NNLO, ... parton distributions are not physical in this scheme. Therefore $\overline{\mathrm{MS}}$ may not be the scheme in which the initial distributions retain their physically motivated shapes (as long known for the photon structure [22]), e.g., for the proton's gluon density at large $x$, see Ref. [23]. Moreover, it seems unclear which positivity bound in particular $g\left(x, \mu^{2}\right)$ has to obey in this scheme, and which NLO partons (if any) are best suited for obtaining estimates from leading-order Monte-Carlo programs [24].

The traditional alternative to $\overline{\mathrm{MS}}$ has been the DIS scheme [25], in which the quark distributions are rendered physical via the structure function $F_{2}$. For the singlet sector the transformation to this scheme is given by

$$
\begin{aligned}
& q_{S}^{\mathrm{DIS}}=q_{S}+\alpha_{\mathrm{s}}\left[c_{2, q}^{(1)} \otimes q_{S}+c_{2, g}^{(1)} \otimes g\right]+\ldots \\
& g^{\mathrm{DIS}}=g-\alpha_{\mathrm{S}}\left[c_{2, q}^{(1)} \otimes q_{S}+c_{2, g}^{(1)} \otimes g\right]+\ldots .
\end{aligned}
$$

Its large drawback is that the second row of Eq. (7) is arbitrary except for the moment $N=2$ fixed by the momentum sum rule. Thus there is nothing physical about the DIS-scheme gluon density especially where constraints are needed most, for very large and for small $x$.

This shortcoming is absent in an interesting old suggestion, the DIS $_{\phi}$ scheme going back (at least) to Ref. [26]. Here also the shape of the gluon distribution is rendered physical via the structure function $F_{\phi}$ of a scalar directly coupling to gluons (such as the Higgs boson in the large- $m_{\text {top }}$ effective theory). The transformation to DIS ${ }_{\phi}$ at $\mathrm{N}^{\mathrm{n}} \mathrm{LO}$ requires the corresponding coefficient functions $c_{\phi, q}^{(n)}$ and $c_{\phi, g}^{(n)}$. Scalar-exchange DIS had to be considered anyway in Ref. [11], and the determination of these coefficient function to order $\alpha_{\mathrm{s}}^{3}$ requires only a minor extension of the published calculations. These functions and possible constraints arising, for example, from the positivity of $F_{\phi}$ will be presented elsewhere.

Now we turn to heavy quarks. For processes at a sufficiently high scale, charm and bottom become effectively light flavours which have to be included in the parton structure of the proton. For most values of $x$ one can disregard a possible non-perturbative 'intrinsic charm' (or bottom) component (which, however, can be relevant at large $x$ for some specific LHC processes [27]). The $\overline{\mathrm{MS}}$ evolution of $\alpha_{\mathrm{S}}[28]$ and the parton densities with a variable number of flavours then proceed via a matching of effective theories for different $n_{f}$. The matching conditions for the parton distributions are especially simple at the heavy-quark mass, $\mu_{\mathrm{f}}=m_{h}$. Denoting the light-quark distributions by $l_{i}$, they up to $\mathrm{N}^{m=2}$ LO read [29]

$$
\begin{array}{ccc}
l_{i}^{\left(N_{\mathrm{f}}+1\right)} & =l_{i}^{\left(N_{\mathrm{f}}\right)}+\delta_{m 2} a_{\mathrm{s}}^{2} A_{q q, h}^{\mathrm{NS},(2)} \otimes l_{i}^{\left(N_{\mathrm{f}}\right)} \\
g^{\left(N_{\mathrm{f}}+1\right)} & =g^{\left(N_{\mathrm{f}}\right)}+\delta_{m 2} a_{\mathrm{s}}^{2}\left[A_{\mathrm{gq}, \mathrm{h}}^{\mathrm{S},(2)} \otimes q_{S}^{\left(N_{\mathrm{f}}\right)}+A_{\mathrm{gg}, \mathrm{h}}^{\mathrm{S},(2)} \otimes g^{\left(N_{\mathrm{f}}\right)}\right] \\
(h+\bar{h})^{\left(N_{\mathrm{f}}+1\right)}= & \delta_{m 2} a_{\mathrm{s}}^{2}\left[A_{\mathrm{hq}}^{\mathrm{S},(2)} \otimes q_{S}^{\left(N_{\mathrm{f}}\right)}+A_{\mathrm{hg}}^{\mathrm{S},(2)} \otimes g^{\left(N_{\mathrm{f}}\right)}\right] .
\end{array}
$$

The results [30] underlying the $q q, h q$ and $h g$ coefficients have been confirmed recently [31].

The matching conditions (8) are included in the above evolution codes and benchmarks. Note that the $\alpha_{\mathrm{s}}^{2}$ NNLO discontinuities were so far ignored in the (published) MRST parton densities. However, they have now been implemented and found to significantly affect the 
cross sections for $W / Z$ production at the LHC [32]. Forthcoming updates of also the NLO distributions will include further significant improvements, e.g., the use of fastNLO [33] for jet cross sections instead of pre-calculated $K$-factor tables.

In general, the calculation of heavy-quark effects on observables is far more involved. We briefly summarize this issue for charm production at HERA, a process which affects the vital extraction of the small- $x$ quark and gluon densities from $F_{2}^{p}$. There are three regimes: For $Q \ngtr m_{c}$ only $u, d, s$ and $g$ act as partons, and charm production can be calculated using the fixed-order massive coefficient functions, presently known to NLO [34]. This framework is usually referred to as the fixed-flavour number scheme (FFNS). At $Q$ ゆ $m_{c}$ all terms with $m_{c} / Q$ are negligible, and $n_{f}=4$ partons - obtained via the matching conditions (8) - can be used with massless four-flavour coefficient functions, a procedure often called the zero-mass variable flavour-number scheme (ZM-VFNS). Finally there is, in general, an intermediate region $Q \gg m_{c}$, where terms with $m_{c} / Q$ are not negligible, but large quasi-collinear logarithms require a resummation via Eqs. (2). Then the $n_{f}=4$ partons have to be used with 'interpolating' coefficient functions for which several prescriptions have been suggested, see refs. [29,35-37]. This is the genuine (or general-mass, GM-) VFNS.

The transition regions between these regimes are process-dependent and tend to lie at higher scales than one might at first expect, something to be kept in mind when using bottom distributions with massless coefficient functions at the LHC. For example, there are strong experimental (see Fig. 9) and theoretical (recall, e.g., Ref. [38]) indications that the FFNS is applicable for the small- $x$ HERA data on $F_{2}^{c}$ at least up to $Q^{2} \gtrsim 100 \mathrm{GeV}^{2}$.

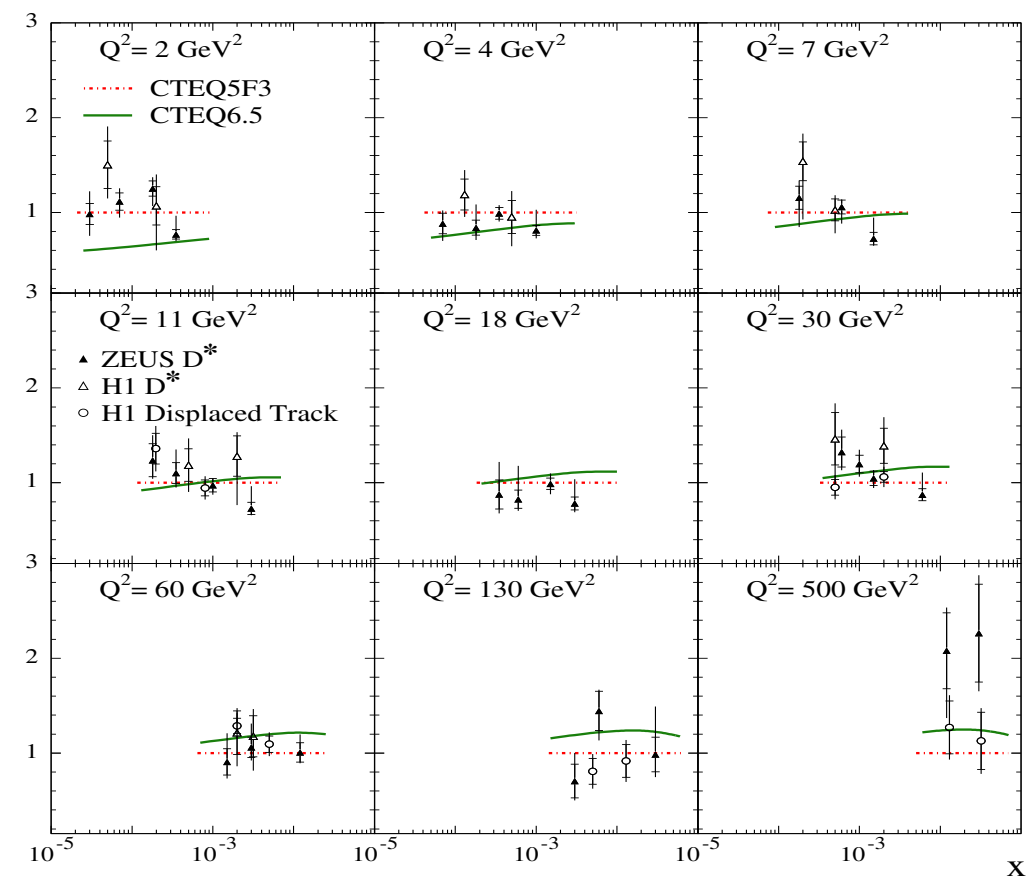

Figure 9: HERA measurements of the charm structure function $F_{2}^{c}$, compared to NLO CTEQ calculations in the fixed [39] and variable flavour-number [37] schemes. All results have been normalized to the former calculation (adapted from Ref. [40]). 


\section{Recent parton analyses and future LHC constraints}

Recently the CTEQ collaboration has published a major update, CTEQ 6.5, of their NLO global fits [37]. A salient improvement is that the mass suppression of the charm contribution to $F_{2}^{p}$ at HERA has finally been included - before the inadequate ZM-VFNS (see above) had been used. The reduced charm component is compensated by larger $u$ and $d$ distributions at small $x$ as illustrated in Fig. 10. This increase leads to larger predictions for the $W$ - and $Z$-production cross sections at the LHC, by about $8 \%$, a shift well outside the uncertainty bands obtained from the previous CTEQ 6.1 sets [41]. It should be noted, however, that both this shift and the similar NNLO result of Ref. [32] mentioned above do not invalidate the widths of the previous error bands. Rather the previous central values should be considered unreliable, as they resulted from fits disregarding well-known theoretical information.
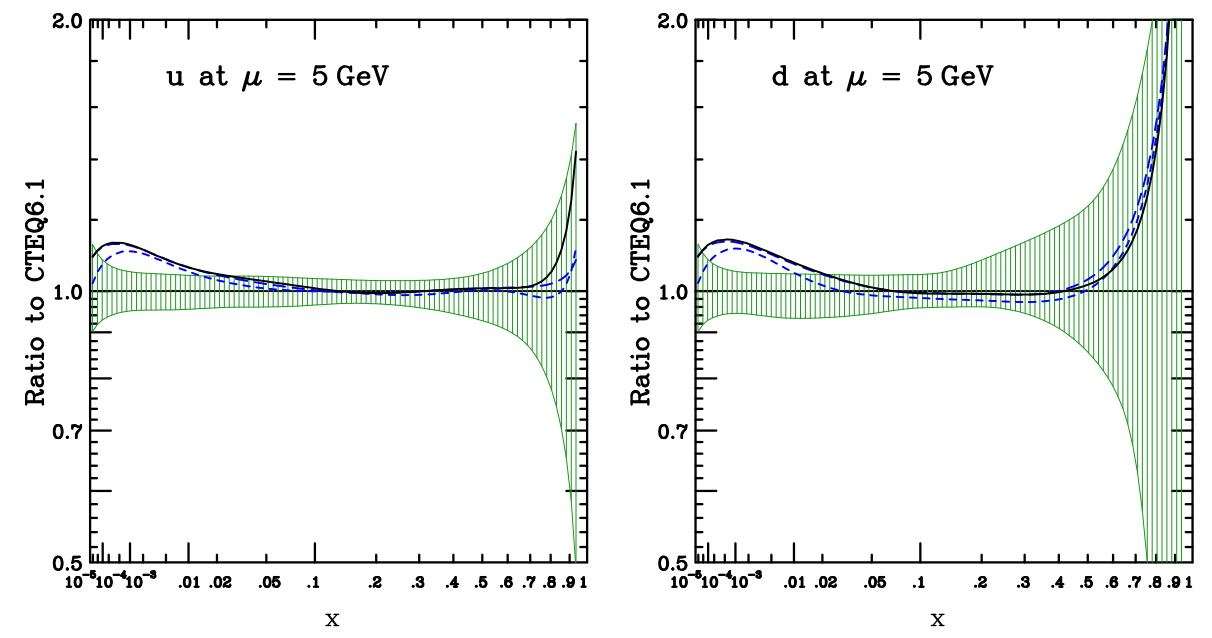

Figure 10: Central $u$ and $d$ distributions of the CTEQ 6.5 fit, normalized to previous results from the same group [41]. Also shown are the estimated error bands resulting from the experimental uncertainties of the data included in the analysis (from Ref. [37]).

In any case, it is important to have at one's disposal several independent sets of parton distributions at each order of perturbative QCD. Until recently, the only NNLO analysis besides those of MRST (now MSTW) was that of Ref. [42], based only on data from deepinelastic scattering. Last year this analysis has been expanded in Ref. [43]: a consistent subset has been included of the available data on Drell-Yan lepton-pair production - note the difference in approach to CTEQ, who are working on their treatment of inconsistent data sets. The NNLO corrections to these cross sections $[44,45]$ are found to be crucial for the fits and, interestingly, as before a rather low value of $\alpha_{\mathrm{s}}\left(M_{Z}\right)$ is preferred, in marked contrast to the recent NNLO fits of MSTW [32].

Usually the initial conditions for Eq. (2) are written in terms of an ansatz, as in Eq. (5) but with more free parameters. The resulting bias is monitored by varying this functional forms as, e.g., in the two dashed curves in Fig. 10. An alternative approach is pursued by the NNPDF collaboration, using neural networks to avoid any such bias. A first analysis of non-singlet structure functions has been performed in Ref. [46], using a new hybrid evolution method combining advantages of the $x$-space and Mellin- $N$ techniques mentioned above. 
Four uncertainty bands for the combination $u+\bar{u}-(d+\bar{d})$ of NLO quark distributions are displayed in Fig. 11. There are many differences between the chosen analyses of Refs. [7, 41, 42, 46], thus it seems difficult to isolate the possible impact of the parametrization bias. It would be interesting to see fits of a reference data set using different approaches to the initial conditions but otherwise identical conditions. In any case, given the precision of the data on the proton structure function $F_{2}^{p}$ and the neutron-proton ratio, for example at $x \approx 0.2$, it seems rather unlikely that the very wide NNPDF band reflects the true uncertainty.

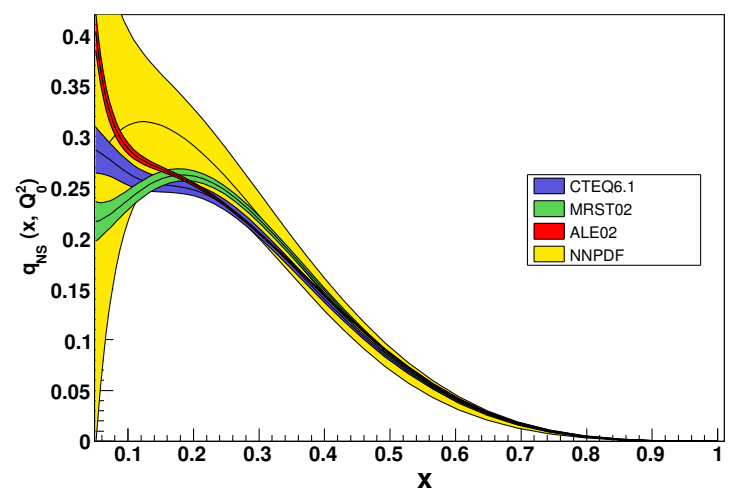

Figure 11: Experimental error bands for the NLO non-singlet combination $q_{\mathrm{ns}}^{+}=u+\bar{u}-(d+\bar{d})$, according to the older fits in Refs. [7,41,42] and the recent NNPDF analysis (from Ref. [46]).

Finally a non-singlet analysis of electromagnetic DIS has been performed in Ref. [47], besides the quark distributions focusing on determinations of $\alpha_{\mathrm{s}}$ up to the $\mathrm{N}^{3} \mathrm{LO}$ of Eq. 3 . This order is accessible outside the small- $x$ region since, as confirmed by Ref. [12], the $\mathrm{N}^{3} \mathrm{LO}$ corrections to the structure function evolution are dominated by the known coefficient functions, see Fig. 20 of Ref. [15]. The results of Ref. [47] for the strong coupling constant read $\alpha_{\mathrm{s}}\left(M_{Z}\right)=0.1134,41 \pm 0.002$ at $\mathrm{N}^{2,3} \mathrm{LO}$, consistent with Ref. [43] but not with Ref. [32]. Obviously more research is required before firm conclusions can be drawn on the uncertainties of the parton densities (as in Fig. 11) and the determination of $\alpha_{\mathrm{s}}$.
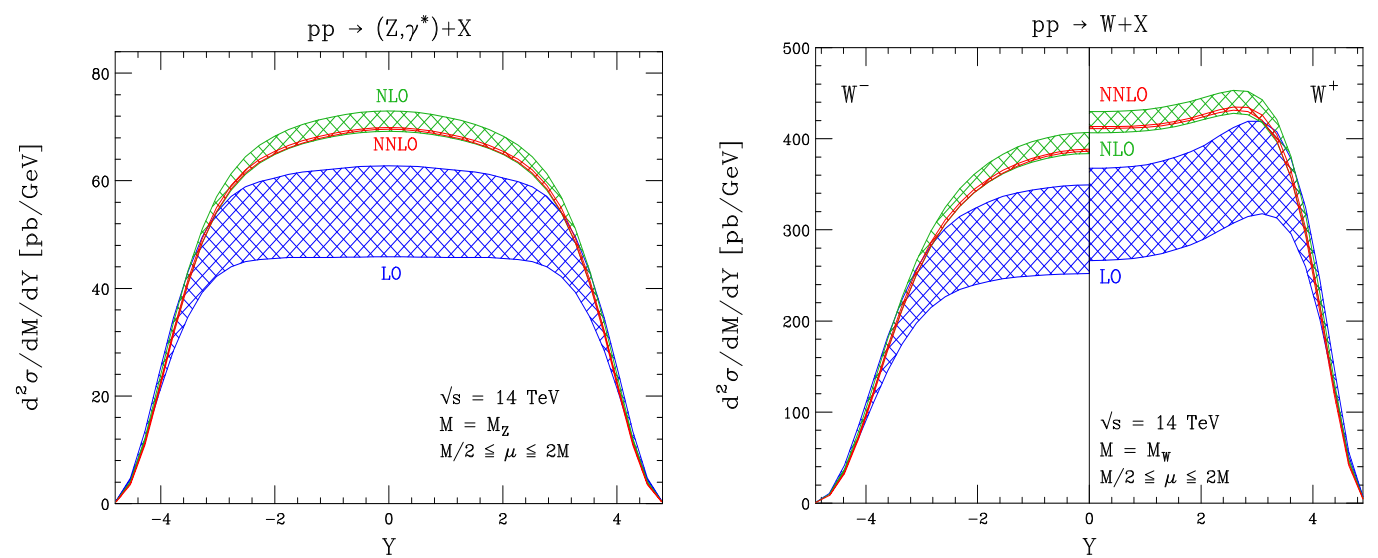

Figure 12: Rapidity-dependent cross sections for gauge-boson production at the LHC, using the partons of Ref. [7]. Shown are the theoretical uncertainty estimates obtained by varying the scale $\mu$ by the arbitrary but conventional factor of two around $M_{W, Z}$ (from Ref. [44]).

The pre-LHC determinations of the parton densities will be improved upon by including reference cross sections measured at the LHC. The 'gold-plated' process of gauge-boson production is illustrated in Fig. 12; see Ref. [48] for a more detailed discussion including 
experimental aspects. The results shown demonstrate the importance of NNLO results even for processes with a far more benign perturbative expansion than the Higgs-production cross section of Fig. 2: It would clearly be impossible to make precision predictions, or perform precision analyses, based on the rough (and non-overlapping) LO and NLO error estimates obtained by varying the renormalization and factorization scale(s). Thanks to the NNLO calculations $[44,45]$, on the other hand, the perturbative uncertainty has been reduced to a level of about $1 \%$, an accuracy unprecedented for hadron-collider cross sections.

\section{Outlook: HERA results for the LHC era}

Precision parton densities and QCD cross sections are required to fully realize the potential of the LHC. For example, a very precise $W$-mass determination with $\delta M_{W} \lesssim 10 \mathrm{MeV}$ seems experimentally feasible, see Ref. [49]. Combined with $\delta m_{\text {top }} \simeq 1 \mathrm{GeV}$ such a result could help to discriminate between, e.g., the standard model and its minimal supersymmetric extension - see the figure (updated from Refs. [50]) shown at the end of the talk [1]. While great progress has been made during the past years, considerable challenges remain.

At the time of this write-up 15 years of data-taking at HERA have ended. Its results will remain indispensable throughout the LHC era, and it is important that also the highluminosity results of the last phase are fully exploited - despite the obvious temptation to move on to, say, LHC Higgs hunting as soon as possible. Moreover, it is highly desirable to preserve important data, e.g., on heavy quarks and jet production, in a manner facilitating detailed re-analyses (as performed for PETRA data in Ref. [51]) a decade from now.

\section{Acknowledgements}

It is a pleasure to thank M. Botje and S. Heinemeyer for providing figures used here and in Ref. [1], and S. Moch and R. Thorne for critically reading parts of the manuscript.

\section{References}

[1] Slides: http://indico. cern.ch/contributionDisplay $\cdot$ py? contribId=4\&sessionId=2\&conf Id=9499

[2] M. Spira, A. Djouadi, D. Graudenz and P.M. Zerwas, Nucl. Phys. B453 (1995) 17, hep-ph/9504378; R. Harlander and P. Kant, JHEP 0512 (2005) 015, hep-ph/0509189

[3] R.V. Harlander and W.B. Kilgore, Phys. Rev. Lett. 88 (2002) 201801, hep-ph/0201206; C. Anastasiou and K. Melnikov, Nucl. Phys. B646 (2002) 220, hep-ph/0207004; V. Ravindran, J. Smith and W.L. van Neerven, Nucl. Phys. B665 (2003) 325, hep-ph/0302135

[4] C. Anastasiou, K. Melnikov and F. Petriello, Nucl. Phys. B724 (2005) 197, hep-ph/0501130

[5] S. Moch and A. Vogt, Phys. Lett. B631 (2005) 48, hep-ph/0508265

[6] V. Ravindran, J. Smith and W.L. van Neerven, Nucl. Phys. B767 (2007) 100, hep-ph/0608308

[7] A.D. Martin, R.G. Roberts, W.J. Stirling and R.S. Thorne, Eur. Phys. J. C23 (2002) 73, hep-ph/ 0110215; Phys. Lett. B531 (2002) 216 hep-ph/0201127

[8] W.L. van Neerven and A. Vogt, Phys. Lett. B490 (2000) 111, hep-ph/0007362

[9] S. Moch, J.A.M. Vermaseren and A. Vogt, Phys. Lett. B606 (2005) 123, hep-ph/0411112

[10] S. Moch, J.A.M. Vermaseren and A. Vogt, Nucl. Phys. B688 (2004) 101, hep-ph/0403192

[11] A. Vogt, S. Moch and J.A.M. Vermaseren, Nucl. Phys. B691 (2004) 129, hep-ph/0404111

[12] P.A. Baikov and K.G. Chetyrkin, Nucl. Phys. (Proc. Suppl.) 160 (2006) 76

[13] J. Blümlein and A. Vogt, Phys. Lett. B370 (1996) 149, hep-ph/9510410

[14] J. Blumlein and A. Vogt, Acta Phys. Polon. B27 (1996) 1309, hep-ph/9603450 
[15] J.A.M. Vermaseren, A. Vogt and S. Moch, Nucl. Phys. B724 (2005) 3, hep-ph/0504242

[16] V.S. Fadin and L.N. Lipatov, Phys. Lett. B429 (1998) 127, hep-ph/9802290; M. Ciafaloni and G. Camici, Phys. Lett. B430 (1998) 349, hep-ph/9803389

[17] W.L. van Neerven and A. Vogt, Nucl. Phys. B588 (2000) 345, hep-ph/0006154

[18] G. Salam, http://projects.hepforge.org/hoppet/

[19] M. Botje, http://www.nikhef.nl/ h24/qcdnum/

[20] A. Vogt, CPC 170 (2005) 65, hep-ph/0408244; http://www.liv.ac.uk/ avogt/pegasus.html

[21] W. Giele et al., hep-ph/0204316 (pages 13 - 22); M. Dittmar et al., hep-ph/0511119 (pages 93 - 102)

[22] M. Glück, E. Reya and A. Vogt, Phys. Rev. D45 (1992) 3986.

[23] A.D. Martin, R.G. Roberts, W.J. Stirling and R.S. Thorne, Phys. Lett. B604 (2004) 61, hep-ph/0410230

[24] J.M. Campbell, J.W. Huston and W.J. Stirling, Rept. Prog. Phys. 70 (2007) 89, hep-ph/0611148; R.S. Thorne, A. Sherstnev and C. Gwenlan, arXiv:0706.2131 [hep-ph] (these proceedings)

[25] G. Altarelli, R.K. Ellis and G. Martinelli, Nucl. Phys. B157 (1979) 461

[26] W. Furmanski and R. Petronzio, Z. Phys. C11 (1982) 293

[27] J. Pumplin, H.L. Lai and W.K. Tung, Phys. Rev. D75 (2007) 054029, hep-ph/0701220; H.L. Lai et al., JHEP 0704 (2007) 089, hep-ph/0702268

[28] K.G. Chetyrkin, B.A. Kniehl and M. Steinhauser, Phys. Rev. Lett. 79 (1997) 2184, hep-ph/9706430

[29] M. Buza, Y. Matiounine, J. Smith and W. L. van Neerven, Eur. Phys. J. C1 (1998) 301, hep-ph/9612398

[30] M. Buza et al., Nucl. Phys. B472 (1996) 611, hep-ph/9601302.

[31] I. Bierenbaum, J. Blümlein and S. Klein, hep-ph/0703285 (Nucl. Phys. B, in press)

[32] A.D. Martin, W.J. Stirling, R.S. Thorne and G. Watt, arXiv:0706.0459 [hep-ph].

[33] T. Kluge, K. Rabbertz and M. Wobisch, hep-ph/0609285 (proceedings of DIS 2006)

[34] E. Laenen, S. Riemersma, J. Smith and W.L. van Neerven, Nucl. Phys. B392 (1993) 162; B.W. Harris and J. Smith, Phys. Rev. D57 (1998) 2806, hep-ph/9706334

[35] A. Chuvakin, J. Smith and W.L. van Neerven, Phys. Rev. D61 (2000) 096004, hep-ph/9910250

[36] R.S. Thorne and R.G. Roberts, Phys. Rev. D57 (1998) 6871, hep-ph/9709442; R.S. Thorne, Phys. Rev. D73 (2006) 054019, hep-ph/0601245

[37] W.K. Tung et al., JHEP 0702 (2007) 053, hep-ph/0611254

[38] A. Vogt, hep-ph/9601352 (proceedings of DIS 96)

[39] H. L. Lai et al., Eur. Phys. J. C12 (2000) 375, hep-ph/9903282

[40] P.D. Thompson, J. Phys. G34 (2007) N177, hep-ph/0703103

[41] J. Pumplin et al., JHEP 0207 (2002) 012, hep-ph/0201195

[42] S. Alekhin, Phys. Rev. D68 (2003) 014002, hep-ph/0211096

[43] S. Alekhin, K. Melnikov and F. Petriello, Phys. Rev. D74 (2006) 054033, hep-ph/0606237

[44] C. Anastasiou, L. Dixon, K. Melnikov and F. Petriello, Phys. Rev. D69 (2004) 094008, hep-ph/0312266

[45] K. Melnikov and F. Petriello, Phys. Rev. Lett. 96 (2006) 231803, hep-ph/0603182

[46] L. Del Debbio et al., JHEP 0703 (2007) 039, hep-ph/0701127

[47] J. Blümlein, H. Böttcher and A. Guffanti, Nucl. Phys. B774 (2007) 182, hep-ph/0607200

[48] A.M. Cooper-Sarkar, hep-ph/0512228 (2005 Les Houches collider-physics workshop)

[49] M. Boonekamp, Acta Phys. Polon. B38 (2007) 2229

[50] S. Heinemeyer et al., Phys. Rept. 425 (2006) 265; JHEP 0608 (2006) 052, hep-ph/0604147

[51] P. Pfeifenschneider et al. [JADE collaboration], Eur. Phys. J. C17 (2000) 19, hep-ex/0001055 